\title{
Improving the safety of chemotherapy prescribing in oncology through the introduction of an assessment proforma
}

\author{
Paula Scullin, Olivia Devlin, Caroline Forde
}

To cite: Scullin P, Devlin 0 , Forde C. Improving the safety of chemotherapy prescribing in oncology through the introduction of an assessment proforma. BMJ Quality Improvement Reports 2017;6:u216501.w7906. doi:10.1136/bmjquality. u216501.w7906

Received 28 October 2016 Revised 2 November 2016

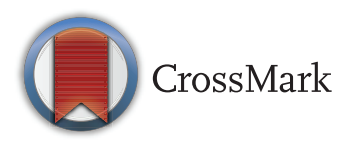

Belfast Health and Social Care Trust, UK

Correspondence to Paula Scullin paula.scullin@ belfasttrust.hscni.net

\section{ABSTRACT}

Chemotherapy remains a high risk treatment with the potential to cause significant patient morbidity and mortality. In the UK the Manual for Cancer Services: Chemotherapy Measures provides national quality measures for essential elements that should be incorporated and documented in chemotherapy assessments.

It was recognised that in the outpatient oncology chemotherapy unit in the Cancer Centre, Belfast City Hospital, Northern Ireland, that the written records of chemotherapy assessments were sub-optimal. At baseline (December 2015) median completion of chemotherapy assessment documentation was only $63 \%$, based on a scoring system incorporating key assessment parameters from the Manual for Cancer Services and Belfast Trust standards for record keeping. A target of median chemotherapy assessment documentation being at least $95 \%$ complete was set.

A paper chemotherapy assessment proforma was developed and introduced over an eight month period, using small tests of change and continuous data collection and feedback. The proportion of chemotherapy assessments documented using the proforma increased, as it was adjusted to be more user friendly and particularly after it started being pre-filed in medical notes.

Increased use of the proforma correlated with improvement in completeness of chemotherapy assessment documentation. From week 29 to project completion (week 33), following proformas being routinely pre-filed and uptake increasing, assessments were on average $97 \%$ complete. Documentation of a patient's performance status, a critical aspect of the assessment, also improved to a median of $99 \%$ over the last seven weeks of the project from a baseline of $88 \%$. The proforma has been positively viewed by staff with $94 \%$ agreeing it promotes safety.

The introduction of a chemotherapy assessment proforma is a simple measure which can result in improved documentation of chemotherapy assessments, including performance status. It also serves as a prompt for safe decision making regarding chemotherapy prescriptions, enhancing the quality of outpatient chemotherapy care being delivered.

\section{PROBLEM}

Advances in cancer treatment have resulted in growing use of chemotherapy treatment (also known as Systemic Anti-Cancer Therapy or SACT): the National Chemotherapy Advisory Group (NCAG) reported a $60 \%$ increase in treatments delivered between 2003 and 2007. ${ }^{1}$

Chemotherapy is delivered in a wide range of patient groups as part of both potentially curative and palliative management plans. In potentially curative treatment regimens maximum tolerated doses of drugs are delivered to achieve greatest efficacy. With palliative treatments the principle aim is to relieve or delay cancer related symptoms and therefore chemotherapy doses are often adjusted to minimise treatment related toxicity.

Chemotherapy has a wide range of potential side effects, which can vary in severity and include nausea, vomiting, mucositis, diarrhoea, fatigue and bone marrow suppression. To reduce the severity of side effects and improve tolerability of treatment adjustments can be made to drug doses, timing of treatment, prophylactic use of antibiotics and growth factors and supportive medications including anti-emetics. It remains however a high risk treatment with the potential for significant, permanent and even life threatening complications. Mortality within 30-days of treatment ranges from $<1 \%->10 \%$ depending on the patient population treated, with higher mortality in less fit patients receiving treatments with palliative intent. ${ }^{2-5}$ Death as a direct consequence of chemotherapy most commonly occurs from neutropenic sepsis or thromboembolic events.

The regional Cancer Centre at Belfast City Hospital in Belfast treats patients from all over Northern Ireland, with an outpatient oncology chemotherapy unit treating approximately 300 patients each week. Patients are assessed prior to each cycle of 
chemotherapy and their treatment prescribed by either a member of the medical team (oncology consultant, specialist trainee, or speciality doctor) or a specialist chemotherapy nurse.

Prior to initiation of this project, prescribers would simply document their assessment 'freehand' in patients' paper medical notes. An annotation of the assessment is then dictated into the patients' electronic oncology notes and an electronic chemotherapy prescription generated.

Reviewing the safety of chemotherapy assessments and prescriptions became a priority in the Cancer Centre in Belfast after a number of Adverse Incidents and patient cases reviewed at the chemotherapy Morbidity and Mortality meeting highlighted areas for improvement. Through review of these incidents similar issues were highlighted to those that were being reported nationally as detailed below.

Although infrequent there were cases where appropriate delays, dose reductions or regimen adjustments had not taken place. There were examples of inadequate grading of performance status (a measure of patients' functional status) and toxicities and also incidents where response assessments had been delayed or not promptly acted on.

It had also been noted that some written notes lacked detail or were difficult to read and as a result there was potential for miscommunication of proposed management plans with pharmacy staff dispensing and nursing staff administering treatment. Reviewing and improving the safety of chemotherapy assessments and their associated written documentation therefore became a priority for the oncology service.

The project aim was to improve the quality of chemotherapy assessments so documentation included at least $95 \%$ of the key parameters drawn from the MCS and Belfast Trust standards for record keeping in oncology patients receiving chemotherapy in the main outpatient chemotherapy unit within a six-month period.

\section{BACKGROUND}

In the UK safety issues regarding chemotherapy prescribing were highlighted in the National Confidential Enquiry into Patient Outcome and Death (NCEPOD) report reviewing patients dying within 30 days of administration of chemotherapy (2008).5 The review found that there was deviation with the standards stated in the Manual for Cancer Services (MCS): Chemotherapy Measures, which aims to provide national quality measures to support the National Cancer Peer Review quality assurance programme and facilitate both clinical and patient centred quality improvement. 6 It concluded that in $49 \%$ of cases reviewed there was room for improvement in the care delivered. For $19 \%$ of patients the decision to start chemotherapy was deemed "inappropriate", in $36 \%$ of patients there was no documentation of toxicities from previous cycles of chemotherapy and in $46 \%$ response to chemotherapy had not been assessed. Following review it was felt a further $13 \%$ of patients should have had a dose reduction, $14 \%$ should have had a treatment delay and in $12 \%$ chemotherapy continued to be delivered despite obvious disease progression. ${ }^{5}$

In response to these concerns NCAG recommended a number of simple measures in their 2009 report "Chemotherapy services in England: ensuring quality and safety". It focused on 'getting the basics right' and included that "standardised processes should be established for recording performance status, investigation results and serious toxicities following a previous cycle of chemotherapy". ${ }^{1}$

The MCS was subsequently updated to reflect the recommendations of NCAG. Essential criteria are clearly defined for what should be recorded for each patient prior to commencing a course of chemotherapy. This includes clear documentation of the intent of treatment, the selected chemotherapy regimen and the intended number of cycles, as well as the investigations required prior to commencing and serially during treatment to assess response. ${ }^{6}$

It also clearly highlights that there should be treatment records for each patient fulfilling the following minimum criteria, prior to each cycle6:

- Results of essential serial investigations applicable to that cycle

- Dose modifications and whether or not they are intended to be permanent

- Cycle (or administration) delays

- New support drugs (e.g. prophylactic growth factors, anti-emetics, laxatives)

- Performance status (graded according to the World Health Organisation system)

- Toxicities following the previous cycle (graded according to the Common Terminology Criteria for Adverse Events (CTCAE))

\section{BASELINE MEASUREMENT}

Data was collected continuously from the outset of this project (December 2015) with an assessment of baseline practice determined from the median of the first ten weeks data. Approximately twenty case notes of patients receiving outpatient chemotherapy were sampled each week for review of the documented chemotherapy assessment. Assessments were scored on a 0-20 point scale, with points awarded for documentation of the following:

- Patient details

- Date and time of the assessment

- Chemotherapy regimen

- Cycle number

- Treatment Intent

- Grade and duration of chemotherapy related toxicities

- Clinical +/- radiological assessment of response 
- Performance Status

- Appropriate management plan including details of modifications required and whether these are intended to be permanent as well as upcoming serial investigations required and whether prescribed within protocol

- Whether the Northern Ireland Electronic Health Care Record (NIECR) had been checked/patient specifically asked about contact with the chemotherapy helpline/GP or hospital

The scoring schema incorporates the key assessment criteria as defined by the MCS (as detailed above), as well as important components of record keeping, as defined by local Belfast Health and Social Care Trust standards, which are based on the General Medical Council guidance in Good Medical Practice (2013). ${ }^{7}$ In addition, the proforma incorporated prompts focused on learning from incident reviews, including prompts to check with the patient and on their electronic care record for hospital admissions or GP contact since last review.

A median score as a percentage was calculated each week as a measure of the completeness of the chemotherapy assessments and written documentation. At baseline chemotherapy assessments were on average $63 \%$ complete (median score approximately 13/20).

There was particular interest in documentation of performance status (PS). Carefully assessing and documenting a patient's PS is a critical aspect of the chemotherapy assessment and significantly influences management, with PS identified as a prognostic factor for chemotherapy-related toxicity and death. $88 \%$ of chemotherapy assessments had a PS documented at baseline.

\section{DESIGN}

A multi-professional quality improvement team (MPQIT) was established from the outset incorporating all those involved in chemotherapy prescribing, dispensing and delivery. It involved medical and non-medical prescribers, pharmacists and chemotherapy nursing staff.

The aim was to improve the safety of chemotherapy prescribing in oncology by ensuring that the written documentation of chemotherapy assessments were at least $95 \%$ complete (score of 19 or greater out of 20 ). It was also agreed that in $100 \%$ of assessments PS should be documented.

A target of 6 months from December 2015 was initially set to deliver the aims of this project. Checklists have consistently been shown to improve safety so it was agreed a paper proforma would be developed encompassing a checklist of the key MCS assessment parameters. 8 The proforma would also be able to incorporate important learning from local 30-day chemotherapy Morbidity and Mortality meetings and incident reviews.

The assessment proforma would aim to ensure the correct information was captured during the consultation, as well as prompting safe decision making and initiation of appropriate management plans. It also sought to improve documentation and communication within the multiprofessional team without being too time consuming or having a detrimental impact on service efficiency.

\section{STRATEGY}

An initial proforma was developed with it agreed that it would take some time to achieve 'buy in' for the project and a proforma that would be universally acceptable and utilised in all chemotherapy clinics, due to significant variation in prescribers' individual practice. It was therefore felt small frequent tests of change, initially with a small group of prescribers on a small number of patients, would be most useful, prior to roll out to larger numbers of prescribers and patients in different clinics.

Weekly PDSA (Plan, Do, Study, Act) cycles were used to make small adjustments and refine the proforma. Changes were made to make it more compact, systematic and user friendly. Furthermore, specific prompts were added, for example with patients that required premedication, had steroids been taken as instructed and had the consent form been signed. Currently version 20 of the assessment proforma is in use (Supplementary File 1).

Data was collected continuously from the outset to provide immediate feedback, with baseline data collected over the first 10 weeks. A minimum of 20 case notes of patients receiving outpatient chemotherapy were sampled each week for review of the documentation from the chemotherapy assessment.

The effects of the implemented changes were predominantly monitored through the use of run charts with results regularly discussed at monthly specialty quality improvement/audit meetings. Continuous feedback was sought from the multi-professional team (predominantly prescribers) informally and also through a written questionnaire approximately six months into the project. The MPQIT met weekly to discuss feedback and agree the next test of change. The multi-professional feedback and frequent meetings were key to maintaining momentum and engagement with users.

Currently the assessment proforma is being used in all oncology chemotherapy clinics in the outpatient unit for all patients. They were originally available loose-leaf in clinic rooms for prescribers to collect and add to the patient's notes but following feedback they are now routinely filed by medical records staff prior to patients attending for assessment. This has helped to make use of the proforma to document the chemotherapy assessment the default.

\section{RESULTS}

Data is presented from Week 1 until Week 33 of the project (just over eight months from when it commenced). Over the course of the project documentation 
from 788 chemotherapy assessments has been reviewed (mean 24 assessments per week).

Proforma use for chemotherapy assessments

The proportion of chemotherapy assessments documented using the proforma increased following amendments based on feedback and increased availability. Progress throughout the project is summarised in Figure 1. From week 28 of the project, when proformas started being routinely pre-filed in medical notes, there has been a steady trend upwards, from $61 \%$ of chemotherapy assessments being documented using the proforma in week 28 to $96 \%$ in week 33 , with a mean of $86 \%$ over weeks $29-33$.

Completeness of chemotherapy assessment documentation

The principle aim of the project was to improve the quality of chemotherapy assessments so documentation included at least $95 \%$ of the aforementioned key parameters drawn from the MCS and Belfast Trust standards for record keeping. The run chart in Figure 2 demonstrates evidence of improvement in completeness of chemotherapy assessment documentation as the project progressed. There was a shift from week 8 of the project with all results collected from that point onwards above the baseline.

Chemotherapy assessments have improved from at baseline being on average 62\% complete (score 13/20) to being consistently $>90 \%$ complete (score 18/20) from week 27 and $>95 \%$ complete (score 19/20) from week 30 . The improvement in completeness of documentation correlates with increased use of the assessment proforma. From week 29-33, when the profroma was being pre-filed and uptake improved, assessments were on average $97 \%$ complete.

Performance Status (PS) Documentation in chemotherapy assessments

There has also been a particular focus on improving documentation of PS to $100 \%$. PS is often referred to as an 'always event' when assessing patients for chemotherapy as it is of such fundamental importance for decision making that an accurate PS should be documented for every patient every time. On eight occasions during the project the $100 \%$ target has been met. PS documentation has improved overall from a baseline of $88 \%$ to a median of $99 \%$ for weeks $27-33$. (Figure 3)

Staff satisfaction questionnaire

Feedback from staff on the assessment proforma (version 20) was sought in May 2016, approximately six months post initial introduction of the template, to try and identify its usefulness and highlight areas for improvement. In total, 34 feedback questionnaires were returned and reviewed (35\% medical staff, $18 \%$ pharmacy staff and $47 \%$ nursing staff including nurse chemotherapy prescribers).

$85 \%$ (29) preferred the new prescribing template in comparison with writing freehand in the medical notes. The remaining $15 \%$ (5) had no preference. 94\% (32) responded that they thought the proforma promoted safety in comparison with writing in the patients' notes.
Figure 1 Proforma use for documentation of chemotherapy assessments

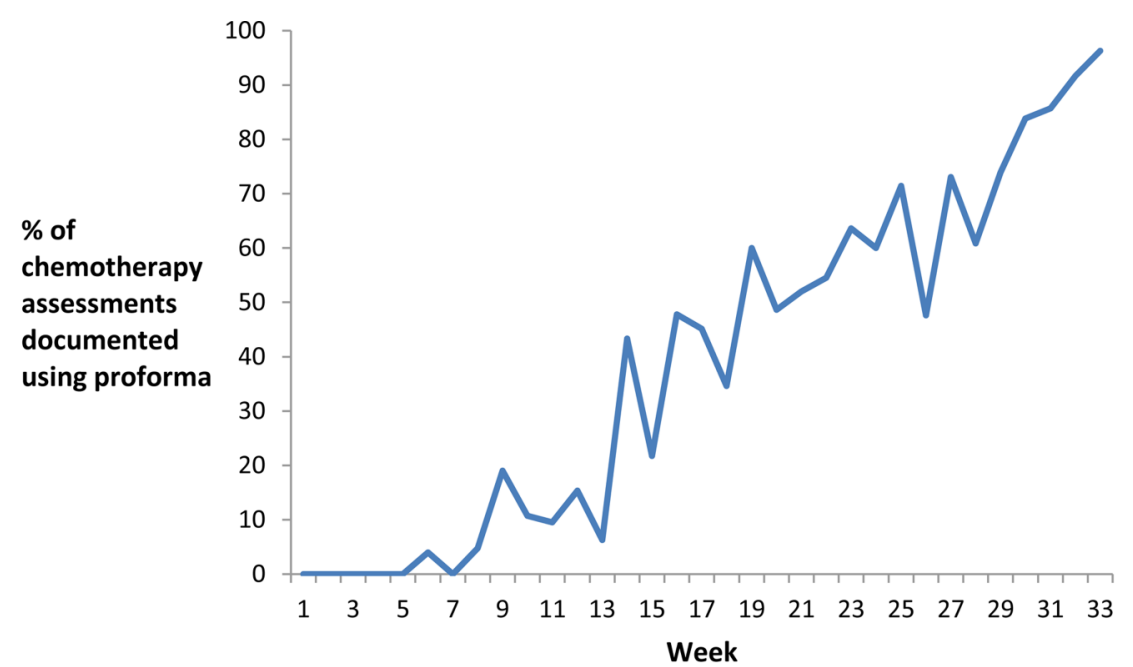

\begin{tabular}{|l|l|}
\hline Key project dates \\
\hline Week 5 & $\begin{array}{l}\text { First version of proforma trialled in single patients in one outpatient } \\
\text { chemotherapy clinic }\end{array}$ \\
\hline Week 8 & $\begin{array}{l}\text { Proforma introduced into second chemotherapy clinic for use in small groups } \\
\text { of patients }\end{array}$ \\
\hline Week 13 & Proforma now in use in all outpatient chemotherapy clinics for all patients \\
\hline Week 27 & Current version of proforma (v20) introduced \\
\hline Week 28 & Proformas routinely pre-filed in medical notes \\
\hline
\end{tabular}




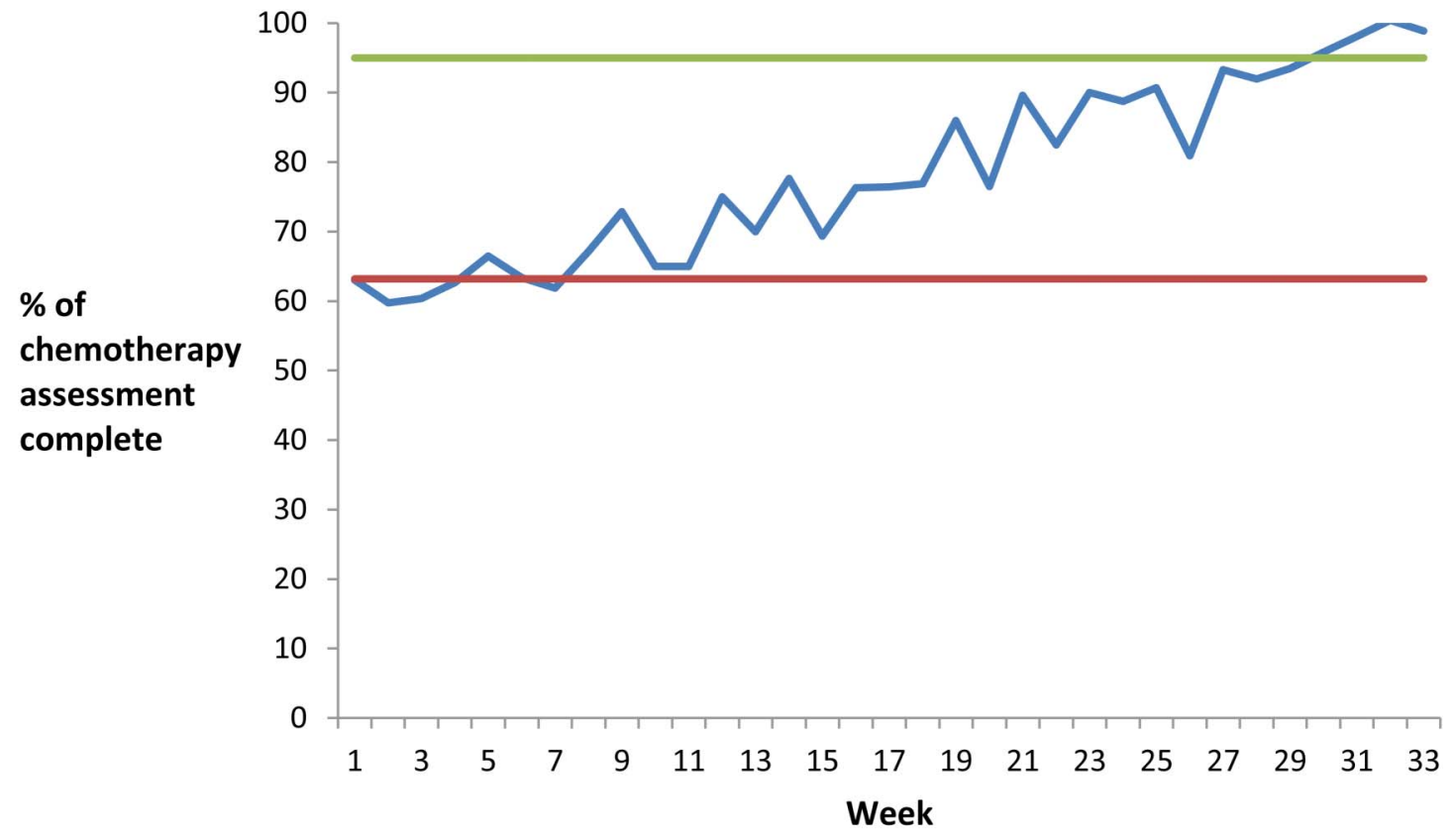

Mean score for weekly data collection $\quad-63.2 \%$ Baseline $\quad-95 \%$ Target

Figure 2 Completeness of chemotherapy assessment documentation

Figure 3 Documentation of Performance Status (PS) in chemotherapy assessments

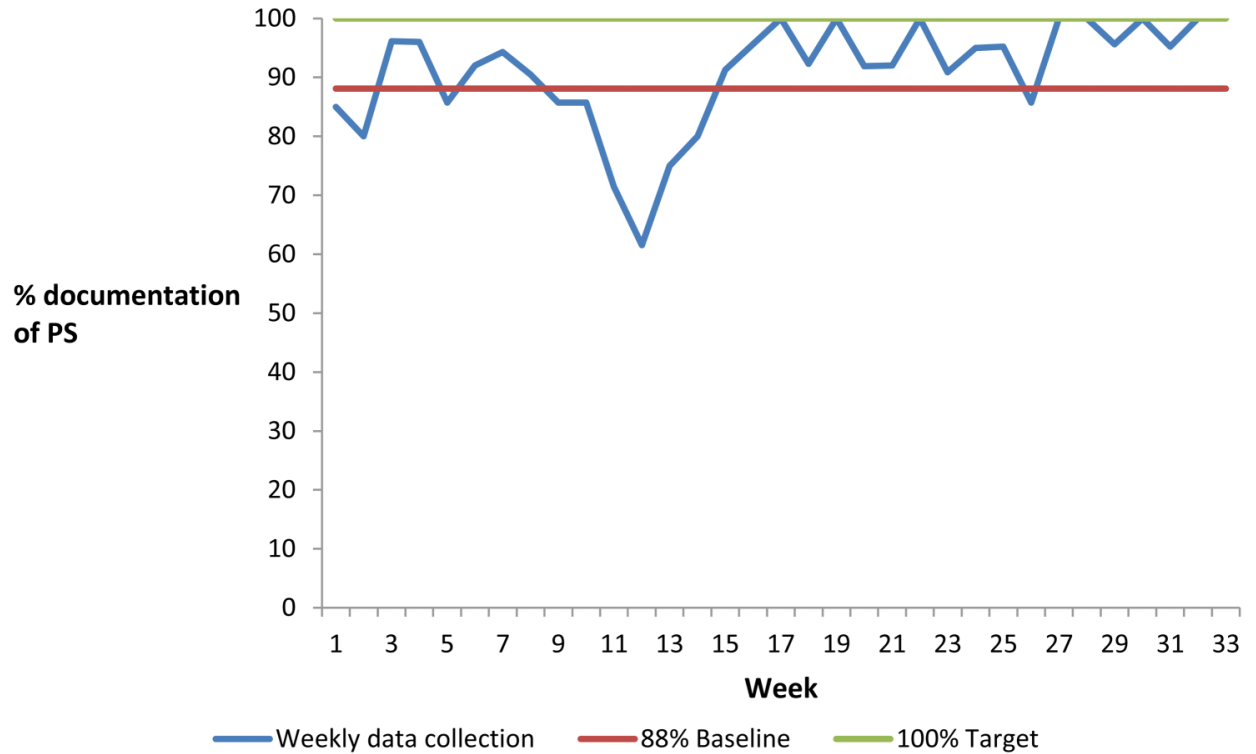

One respondent felt it didn't promote safety and one failed to answer the question.

Staff were given the opportunity to comment on what they liked about the template. Common themes included that it was a useful document which had a clear, straight forward, systematic structure which prompted documentation of the important issues and was quick to complete. Nursing staff in particular found it easier to identify prescribers as medical staff are prompted to print their names.

Staff were also provided with the opportunity to comment on aspects they importantly didn't like.
Common themes included that proformas were not being filed or incorrectly filed and had the potential to go missing. Some commented they found it more time consuming and that they disliked too many tick boxes. Others commented that the proforma was not being fully completed by all prescribers and in particular that dates and names of staff were not always recorded.

Suggestions as to how the chemotherapy assessment could be improved were also sought. The most frequent suggestion was that the proforma should be pre-filed in the medical notes. This was extremely valuable feedback as sometimes due to lack of availability of proformas in 
clinic rooms users had simply reverted to writing in the notes again. As a result of this feedback staff in medical records now file a proforma in the medical notes prior to patients attending clinic and this has had a significant impact on the proportion of proformas being completed.

\section{LESSONS AND LIMITATIONS}

This was a challenging and ambitious project to undertake as it sought to introduce a different approach for prescribers who had different or well established ways of documenting chemotherapy assessment. Progress particularly initially was slow with resistance to change noticeable amongst some staff members. A committed and enthusiastic team leading the project was essential to persevere and make progress.

Immediately starting to test the proforma on a small scale was a useful way to kick start the project with staff that were engaged and helpful. It was evident that it was not until the proforma was being used in 'real practice' that its limitations were revealed. When the proforma was being rolled out across different chemotherapy clinics it was imperative staff were familiar with it but even more importantly aware of the rationale and aims of the project, so they could understand why they were being asked to do something differently.

Obtaining honest feedback and suggestions from the whole multiprofessional team remains an essential component of analysing implemented change. Facilitating written, anonymous feedback through a questionnaire was a particularly beneficial tool for capturing feedback from a wide range of staff. It provided an opportunity for those who may not normally express their opinions at meetings to contribute and allowed for more thoughtful and candid comments. This approach for assessing satisfaction with implemented changes will definitely be utilised in other quality improvement work being undertaken in our oncology service.

This project remains at an early stage and will require a long term commitment from the team involved to ensure its longevity. The detailed information required during a chemotherapy assessment lends itself well to a proforma and we were eager to share our template, which may be useful to other centres who like us document assessments in patients' paper medical notes. It is appreciated however that some centres already use only electronic platforms for chemotherapy assessment documentation but this project nevertheless demonstrates the strength of checklist components being incorporated into proformas rather than just freeform annotations.

\section{CONCLUSION}

The development of a chemotherapy assessment proforma, using small tests of change with continuous data collection and feedback, has resulted in a version that is positively viewed and widely used within our Cancer
Centre's outpatient oncology chemotherapy unit. Increased use of the proforma has correlated with improvements in overall completeness of assessment documentation, including improved documentation of PS to $99 \%$ from a baseline of $88 \%$.

It is now planned to share the learning and results from this project regionally with the other cancer units in Northern Ireland. Additional training is being developed to familiarise staff, especially new prescribers, with completion of the proforma. A nursing administration checklist is currently also being developed to complement and strengthen the clinician's proforma, to further enhance safe chemotherapy prescribing and improve the quality of care being delivered.

Acknowledgements This quality improvement project was completed as part of a Scottish Patient Safety Fellowship Programme under the guidance of Clinical Lead Ros Gray.

With thanks to the MPQIT in the Cancer Centre, Belfast City Hospital who assisted with this project: Debbie McKelvey (Lead Nurse for SACT BHSCT), Elaine Shaw (Breast cancer CNS and NMP), Catherine Goudy (Oncology and Haematology Specialist Pharmacist), Nicki Delaney (Deputy Ward Manager, Oncology Day Unit), Una Rodgers (Medical Records Supervisor) and Aine Mckeown (Management Intern).

With thanks also to the entire medical, nursing, pharmacy and medical records staff in the outpatient chemotherapy unit for their support and engagement with this project.

Declaration of interests No conflicts of interest to declare.

Ethical approval Ethical approval for this project was not required due to it being a quality improvement project aimed at improving the safety of chemotherapy prescribing in the Belfast Health and Social Care Trust.

Open Access This is an open-access article distributed under the terms of the Creative Commons Attribution Non-commercial License, which permits use, distribution, and reproduction in any medium, provided the original work is properly cited, the use is non commercial and is otherwise in compliance with the license. See:

- $\mathrm{http}: / /$ creativecommons.org/licenses/by-nc/2.0/

- http://creativecommons.org/licenses/by-nc/2.0/legalcode

\section{REFERENCES}

1. National Chemotherapy Advisory Group. Chemotherapy Services in England: Ensuring quality and safety: a report from the National Chemotherapy Advisory Group. 2009. http://ncat.nhs.uk/sites/default/ files/NCAG\%20Report.pd

2. O'Brein ME, Borthwick A, Rigg A et al. Mortality within 30 days of chemotherapy: a clinical governance benchmarking issue for oncology patients. Br J Cancer, 2006, 95: 1632-36.

3. Khoja L, McGurk A, O'Hara C et al. Mortality within 30 days following systemic anti-cancer therapy, a review of all cases over a 4 year period in a tertiary cancer centre. Eur J Cancer 2015; 51: 233-40.

4. Zdenkowski N, Cavenagh J, Ku YC et al. Administration of chemotherapy with palliative intent in the last 30 days of life: the balance between palliation and chemotherapy. Intern Med J 2013; 43: 1191-98.

5. National Confidential Enquiry into Patient Outcome and Death (NCEPOD). For better, for worse? A review of the care of patients who died within 30 days of receiving systemic anti-cancer therapy. 2008. http://www.ncepod.org.uk/2008report3/Downloads/SACT_ report.pdf

6. NHS England, National Peer Review Programme. Manual for Cancer Services: Chemotherapy Measures Version 1. 2014. www.cquins. nhs.uk/.../measures/Chemotherapy_April2014.pdf

7. General Medical Council. Good Medical Practice.2013 http://www. gmc-uk.org/guidance/good_medical_practice.asp

8. Haynes et al. A Surgical Safety Checklist to Reduce Morbidity and Mortality in a Global Population. N Engl J Med 2009; 360: 491-99. 